\title{
Challenges and Opportunities in the Multivariate Analysis of 3-D Spectral Images
}

\author{
Michael R. Keenan ${ }^{1}$ \\ 1. Unaffiliated, 8346 Roney Road, Wolcott, NY, 14590, USA
}

Multivariate statistical methods have long proven powerful for extracting chemical information from spectroscopic images. The premise underlying this application of multivariate techniques is that the observed spectrum at any point can be described as an additive linear mixture of a limited number of base spectra or "pure components". Recent interest has focused on applying these methods to data sets comprising full spectra acquired from samples in three spatial dimensions. Some examples include EDS tomographic images [1], ToF-SIMS imaging depth profiles [2] and atom probe tomography datasets [3]. Besides the obvious computational challenges posed by increasingly large data sets in 3-D, are more subtle concerns. Consider, for instance, a 10-pixel inclusion in a matrix whose characteristic size is 100 pixels. Spectra arising from the inclusion would account for about $10 \%$ of the total in a line scan, $1 \%$ in an image but only $0.1 \%$ of the voxels in a 3-D dataset. It appears that finding the proverbial needle is likely to be particularly difficult in a three-dimensional haystack. It will be shown, however, that Independent Component Analysis (ICA) is well suited for identifying minor components in a dataset. Furthermore, it will be shown that sparseness can be induced in a dataset via a wavelet transform, allowing ICA to extract more physically reasonable representations of all components, both major and minor.

The first step in multivariate analysis is often to reduce the problem size. This is typically accomplished by decomposing a data matrix into spatial and spectral factors via Principal Component Analysis (PCA). Such factor models, however, are well known to suffer a "rotational ambiguity". That is, an infinity of factor models exist that will fit the data equally well. Relatively unique and easily interpretable factors are obtained by restricting the models to conform to known or likely characteristics of the data. For counting experiments, the spectra and abundances are fundamentally non-negative, a fact that is exploited by multivariate curve resolution $[1,2]$. If, on the other hand, samples consist of discrete chemical phases, standard methods such as Varimax rotation can be used to resolve spatially simple models [4].

Here, ICA will be presented as yet another factor rotation method. ICA assumes that the components are statistically independent. Roughly, this means that having complete knowledge about one component conveys little information about any others. Knowing everything about the needle, for instance, says nothing about the haystack, overall. ICA will be illustrated with a previously published 3-D EDS dataset, and full experimental details can be found in [1]. The sample consists of localized corrosion on a $\mathrm{Cu}$ substrate, with a protective Pt overlayer. PCA and ICA are compared in columns (a) and (b) of the figure for one slice through the sample. Only components relevant to the copper surface and corrosion products are shown. Both PCA and ICA yield factors that are abstract in the sense that they display large negative features that are physically implausible. The abstract natures of these factors are a direct result of violating the assumptions underlying the respective methods. The ICA picture is arguably easier to interpret, however, as the spectral components are generally simpler. This is most noticeable for a small region with $\mathrm{Si}$ and $\mathrm{O}$ that is isolated by ICA, but requires 4 principal components, 1 and 3-5, to fully describe it.

While ICA does a good job of extracting the sparsely distributed components from the spectral image, extracting the major components presents a greater challenge. Both PCA and ICA represent the spatially distinct $\mathrm{Au}$ and $\mathrm{Pt}$ distributions as mixtures, factors 5 and 6 in the figure, rather than as pure elements. 
One way to improve the performance of ICA, in this regard, is to induce sparseness in the data. This can be accomplished with a wavelet transform. For typical images, wavelet transforms have the ability to concentrate the information in the image into relatively few large-magnitude coefficients, leaving many small ones that often can be discarded as inconsequential ("decimated"). Column (c) in the figure shows the results of ICA applied to undecimated wavelet-transformed data directly in the transform domain. After applying the inverse transform to the spatial factors, we note that all of the corrosion-related components are plainly resolved with spectral factors that are physically plausible. Notably, Au and Pt are no longer commingled, but rather, are represented as relatively pure elements (Pt not shown).

\section{References:}

[1] PG Kotula, MR Keenan and JR Michael, Microsc. Microanal. 12 (2006), p. 36.

[2] VS Smentkowski, et al, Anal. Chem. 20 (2007), p.7719.

[3] MR Keenan, et al, Microsc. Microanal. 17 (2011), p. 418.

[4] MR Keenan, Surface and Interface Analysis 41 (2009), p. 79.

(a) PCA

(1)

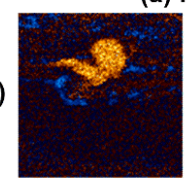

(2)

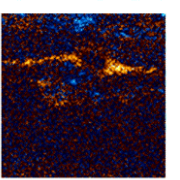

(3)

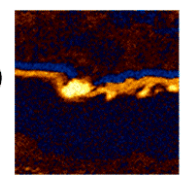

(4)

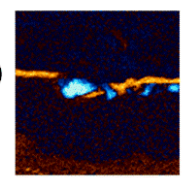

(5)

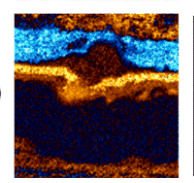

(6)

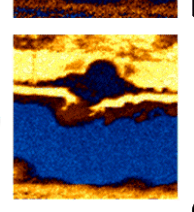

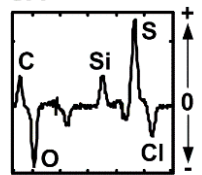
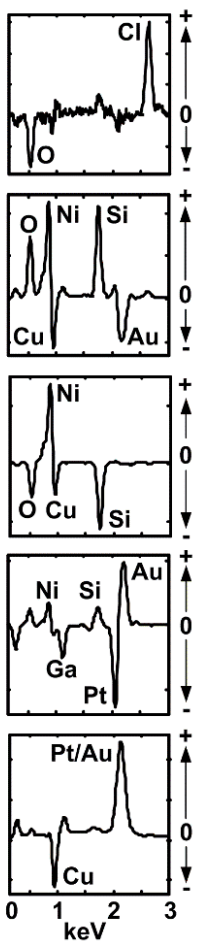

(b) ICA
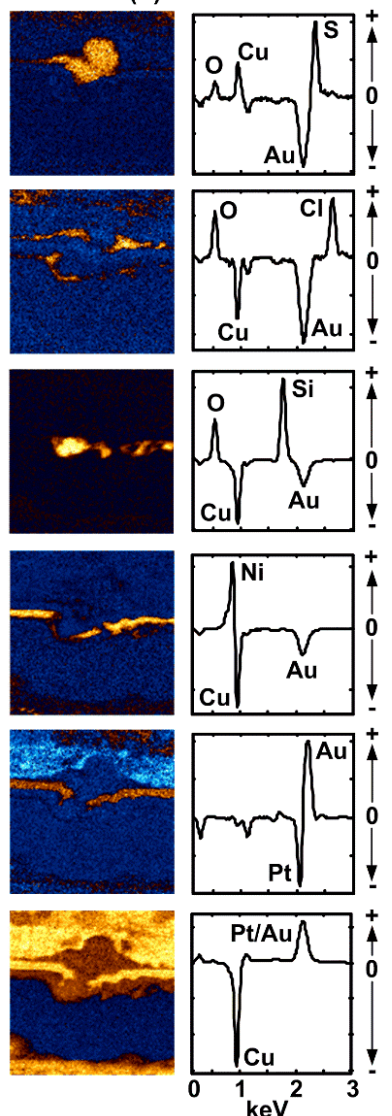

(c) Wavelet/ICA
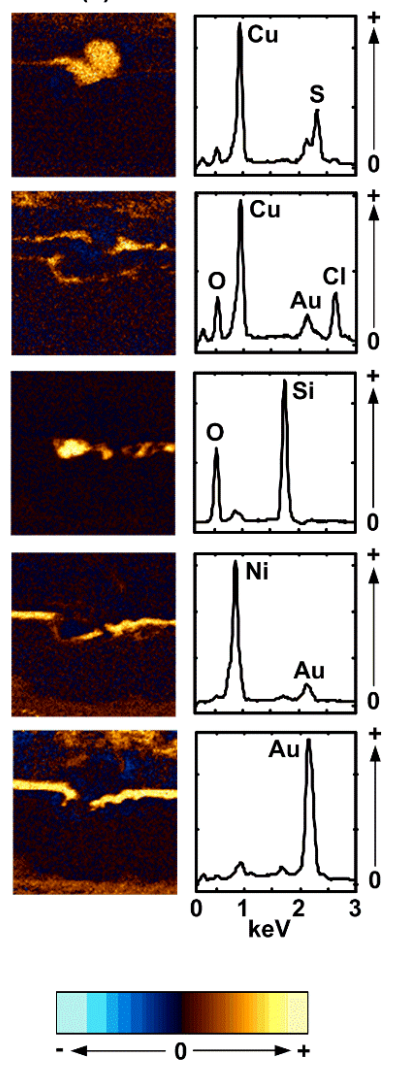

Figure 1. Spectral factors, and spatial factors for one slice, of a 3-D spectral image of corrosion on a copper substrate. Principal components and independent components are shown in (a) and (b), and (c) shows independent components derived from undecimated haar-wavelet-transformed spectral data. 